\title{
GERBERTO DE AURILLAC Y SU TIEMPO Nota sobre el Congreso internacional de Vic (noviembre de 1999) sobre el tema
}

En la catalana ciudad de Vic, a unos $60 \mathrm{~km}$. al norte de Barcelona, se inauguraba el miércoles $10 \mathrm{de}$ noviembre de 1999 un Congreso internacional sobre el papa del año 1000, Silvestre II, con ocasión del milenario de su acceso a la sede pontificia, acaecido en abril del 999. Destacados historiadores y otros especialistas, así como estudiantes de la Universitat de Vic y diversas personas interesadas en el tema -un conjunto de cerca de 200 asistentes - protagonizaron o siguieron las sesiones académicas, que concluyeron el viernes 13 , al acabar el cuarto día, en Ripoll, población situada a otros $30 \mathrm{~km}$. al norte de Vic. Organizado por las universidades públicas catalanas (la de Barcelona, la Autónoma de Barcelona, la Pompeu Fabra [también de Barcelona], la de Girona, la de Lleida, la Rovira i Virgili [de Tarragona] y la de Vic), con la colaboración del Institut d'Estudis Catalans, del Arxiu Episcopal de Vic y de la misma Generalitat de Catalunya, el Congreso se articulaba en forma de 16 ponencias, y contaba además con la presentación de 36 comunicaciones. Sorprendentemente (no es esto lo que suele acontecer en ocasiones como ésta), las Actas del Congreso estuvieron a disposición de los asistentes ya en el segundo día del mismo. ${ }^{1}$

El título del Congreso era: «Gerberto de Aurillac y su tiempo. Cataluña y Europa a fines del primer milenio». Aparte de la inaugural, a que enseguida nos referiremos, el resto de ponencias y las comunicaciones se agrupaban en seis núcleos temáticos (según su orden en las Actas, distinto del del programa del Congreso): 1 . Cataluña y el mundo hace mil años; 2 . Testimonios materiales en torno al año mil; 3 . Las lenguas en torno al año mil: coexistencia y evolución; 4. La enseñanza en el año mil; 5. La ciencia y la cultura: cristianos, judíos y musulmanes a finales del primer milenio; y 6 . El monacato y el papado en el año mil.

El agotador ritmo de las sesiones tuvo un doble y agradable descanso: hubo, a mitad del Congreso, una visita al pequeño monasterio benedictino de Sant Pere de Cassarres — de los primeros años del s. XI, recién restaurado, y situado en el incomparable paraje (aquel día, impregnado de lluvia) de un perdido y escarpado meandro del Ter a su llegada al actual pantano de Sau-, en cuyo inmenso y frío templo románico se ofreció un cálido concierto de melodías medievales, al que siguió luego una comida de todos los congresistas; y hubo, el último día, otra visita al gran monasterio de Santa María de Ripoll, donde tuvo lugar la ponencia que servía de colofón del Congreso. Fundado en el 879 , antes de un siglo este monasterio, también benedictino, contaba en su Escritorio con una impresionante colección de códices manuscritos que sirvieron al joven monje Gerberto, entre el 967 y el 970, para hacerse con saberes que en la occitana Alvernia, su tierra de origen, nunca hubiera podido adquirir.

\section{LECCIÓN INAUGURAL: EL ESTADO DE LA CUESTIÓN GERBERTIANA}

Tras los parlamentos de rigor — del Rector de la Universitat de Vic y de la coordinadora del Congreso-_, inauguraba éste el mejor especialista en Gerberto, el historiador francés Pierre Riché, abordando los últimos «Dos siglos de estudios gerbertianos». ${ }^{2}$ Su exposición magistral se estructuró según cuatro momentos: 1)

1 Actes del Congrés Internacional: Gerbert d'Orlhac i el seu temps; Catalunya i Europa a la fi del primer mil.leni (Vic-Ripoll, 10-13 de novembre de 1999), Eumo Editorial (Documents 31), Vic 1999, 888 p.

2 Cf. o. c., p. 23-42: «Deux siècles d'études gerbertiennes». 
Gerberto en los s. XIX y XX; 2) Las fuentes de nuestro conocimiento del mismo; 3) Las etapas de su carrera; y 4) Sus etapas intelectuales. De sus contenidos parece inexcusable ofrecer el siguiente resumen. ${ }^{3}$

[1] Olvidado durante centurias, Gerberto fue objeto de atención en el s. XVI por los galicanos y los protestantes franceses, pues todos ellos creían descubrir, en las Cartas de aquél —que sin duda son la fuente fundamental para el conocimiento de su vida y que él mismo seleccionó y ordenó para que fueran conservadas-, argumentos contra el papado; en los dos siglos siguientes, dichas cartas suscitaron asimismo el interés de los benedictinos de San Mauro y de los galicanos moderados, pero hay que esperar a mediados del s. XIX para que las obras de Gerberto fueran publicadas en edición mínimamente crítica. ${ }^{4} Y$ son los últimos años de dicho siglo - acaso por influencia de lo anterior - los que, junto a una nueva edición de las cartas (hecha por J. Havet en 1889), conocen la primera gran eclosión de estudios no sólo sobre Silvestre II, presentado como «un papa filósofo», sino también sobre las obras científicas de Gerberto anteriores al acceso de éste al pontificado, ${ }^{5}$ estudios que se suceden intermitentemente con posterioridad, ya en el s. XX. ${ }^{6}$ Pese a la solemne celebración que en julio de 1938 hubo en Aurillac de los mil años del nacimiento de Gerberto, la Segunda Guerra Mundial impedía cualquier nueva eclosión de estudios gerbertianos. Acabada dicha guerra, destacados historiadores franceses empezaron a comprender que el s. X no era sólo un «siglo de hierro»; ${ }^{7}$ y dejaron que aquella nueva eclosión coincidiera con las dos últimas décadas de nuestro siglo: un tanto imperfecta, al principio (el simposio de 1983 conmemorativo de los mil años del desempeño por parte de Gerberto del cargo de abad en Bobbio no recogía en sus actas — pese al pomposo título de las mismas - la importancia de la obra gerbertiana), ${ }^{8}$ dicha eclosión no deja de tener al mismo P. Riché como autor destacado. ${ }^{9}$ Diversos congresos dan, por lo demás, elocuente testimonio del alcance de la misma. ${ }^{10}$

3 Parece asimismo oportuno presentar también, en notas, algo así como un extracto del aparato crítico de la ponencia, a modo de «puesta al día» bibliográfica que pueda ser de utilidad para el lector interesado al respecto.

4 A. Olleris, Oeuvres de Gerbert pape sous le nom de Sylvestre II, Clermond-Ferrand, París 1867. No fue ajena a esta edición la sustanciosa polémica que, con ocasión del concilio Vaticano I, sostuvieron P.F. LAUSSER (Gerbert, étude historique sur le Xe siècle, Aurillac 1866; reimpr., Ginebra 1977), por un lado, y E. MorIN ( «Le concile de Saint Basle. Récits du Xe siècle», en Mémoires de la Société Académique du Maine et Loire, 1868, p. 90-139) y P. COLOMBIER («Vie de Gerbert», en Etudes, 1869, p. 83-110 y 248-279, y «Défense de Gerbert», id., p. 604-617), por otro, negando sin razón el primero la autenticidad de una parte de la correspondencia gerbertiana y defendiéndola los segundos, que se esforzaban asimismo en explicar la actitud de Gerberto respecto del concilio de Saint-Basle.

5 Recuérdense, entre otras, las siguientes aportaciones: R. ALLEN, «Gerbert, pope Silvester Il», en English Historical Review, 1892, p. 625-668; A. GRAFF, «La legenda di un pontefice», en Miti, legende e superstitioni del Medio oevo, Turín 1893, t. II, p. 3-48; F. PICAVET, Gerbert un pape philosophe d'après l'histoire et la légende, París 1897; y C. LuX, «Papst Silvester II», Breslau 1898.

6 Véanse no sólo la voluminosa obra del duque de LA SALLE DE RoCHEMAURE, Gerbert-Silvestre II. Le savant, le faiseur de rois, le Pontife —cuya aparición, en 1914, coincidía bien inoportunamente con el inicio de la Primera Guerra Mundial一, sino sobre todo los espléndidos «Studien zu Gerbert von Aurillac», de M. UhrLIRZ, publicados en el Archiv fur Urkundenforschung, 1930, p. 391-422, y 1935, p. 437-447.

7 Véanse J. LeFlon, Gerbert: humanisme et chrétienté au Xe siècle, Abbeye de St. Wandrille 1946; H. Focillon, L'an mil, París 1952; L. GRODECKI, L'architecture ottonienne, París 1958 y Le siècle de l'an mil, París 1973; «Mirabilia mundi. Essai sur la personnalité d'Otton III», en Cahiers de civilisation médiévale, 1963, p. 297-313 y 454-476; E. Olurvier, Otton III empereur de l'an mille, Lausana 1969; G. Duby, L'an mil, París 1967; y PH. WolfF, L'éveil intellectuel de l'Europe, París 1971.

8 Cf. Gerberto. Scienzia, storia e mito. Atti del Gerberti Symposium, Bobbio 1985.

9 Cf. F. TRistram, Le coq et la louve. Histoire de Gerbert et de l'an mille, París 1982; y P. RichÉ, Gerbert d'Aurillac, le pape de l'an mil, París 1987; «Nouvelles recherches sur les lettres de Gerbert d'Aurillac», en Comptes rendus des séances de l'Académie des Inscriptions et Belles Lettres 1987, p. 575-585; «Introduction» a La correspondance de Gerbert d'Aurillac, ed. Les Belles Lettres, París 1993; Les grandeurs de l'an mille, París 1999.

10 Se han celebrado, en Aurillac mismo, un coloquio - cuyas actas, intituladas Gerbert l'Européen, publicaba en 1997 la sociedad La Haute Auvergne-, en 1996, y unas jornadas -la publicación de cuyas actas prepara la misma sociedad-, en 1999, año en el que también tenía lugar el Congreso del que da cuenta la presente nota y que se abría con la ponencia de Riché. 
[2] Ya hemos aludido a las Cartas de Gerberto como fundamentales para el conocimiento de su vida." Pero fuente no menos decisiva al respecto, siguiendo una vez más a Riché, es la Historia de Francia que nos dejó el monje Riquerio de Reims, ${ }^{12}$ discípulo de Gerberto, Riquerio no ha dejado de tener su lugar en la biliografía reciente. ${ }^{13}$ Las lagunas que puedan presentar los capítulos (de los libros III y IV de la obra) centrados en la figura del maestro --por ejemplo, la de la no inclusión in extenso de los textos del concilio de SaintBasle y correspondiente omisión de lo que en ellos había de más hostil al papado- deberán ser subsanadas por el estudio comparativo de los manuscritos en que nos han llegado las fuentes en cuestón. ${ }^{14} \mathrm{El}$ de los manuscritos es, por lo demás, un campo abierto de investigación, por la cantidad que de los mismos —en su mayoría de los s. XI y XII- disponemos pero que no han sido aún estudiados como merecen. ${ }^{15}$

[3] Nueve son las etapas que Riché distinguió en la carrera de Gerberto. ${ }^{16} \mathrm{Y}$ en cada una de ellas se refería a uno u otro de los puntos más destacados de las mismas y al estado actual de la investigación sobre ellos.

1. El monje de Aurillac. Descartadas las leyendas locales que le hacen hijo de Beliac o Saint Simon, cabe decir que no conocemos el lugar de nacimiento de Gerberto: un nombre, por lo demás, entonces común en el sur de Francia y en Cataluña. ${ }^{17}$ Sus padres le confiaron a la célebre y cercana abadía de Saint Géraud, en Aurillac, reformada por Odón de Cluny. ${ }^{18}$ De su formación allí como monje benedictino le quedará una huella indeleble. ${ }^{19}$

2. Estudiante en Cataluña. «Con el consentimiento de los monjes» de Aurillac (en palabras de Riquerio), Gerberto marcha a Ripoll a ampliar sus estudios en el Escritorio del monasterio. ${ }^{20}$ Aunque no es muy claro el papel que Vic pudo representar al respecto, lo cierto es que el joven estudiante estableció además sólidas amistades: con el por entonces abad de Cuixà, a quien en 985 dirigirá su carta 28; con Miró Bonfill, obispo de Gerona, ${ }^{21}$ y con el archidiácono de Barcelona Llobet. ${ }^{22}$ Igualmente incuestionable es su relación con el conde Borrell, ya que con el tiempo desempeñará para con él la función de intermediario - según confirman su carta 70 y el testimonio de Riquerio- ante Hugo Capeto, siendo secretario de éste. En fin, ya papa, no dejará de conceder bulas a sus amigos catalanes para defenderlos de sus adversarios. ${ }^{23}$

11 La antigua versión francesa de E. DE BERTHÉLEMY (París-Lyon 1868) y la mencionada edición latina de J. Haver (de 1889) de la correspondencia gerbertiana tienen su réplica más reciente en la edición de 1993 a cargo de P. RicHÉ y J.P. CaLLu (cf. supra nota 8), en latín y francés. De las Cartas hay también una traducción en inglés, de H.P. LaTTiN (Nueva York 1961), y otra en italiano, de M.G. PANvinI CARCIOTto: en Quaderni di Filologia Medioevale I (1980), fasc. 1, p. 155-233 y fasc. 2, p. 79-211, y IV (1983), fasc. 4, p. 117-241.

12 Richer, Histoire de France, ed. y trad. francesa de R. LATOUCHE, París 1930 y 1937, reed. 1964 y 1967.

13 Cf. H.H. KoRTum, Richer von Saint-Remi. Studien zu einem Geschichtsschreiber des 10. Jahrhunderts, Stuttgart 1985; y M. SoT, «Richer de Reims a-t-il écrit une «histoire de France»?», en Histoire de France, Historiens de la France (ed. Y.M. Bercé y Ph. Contamine), París 1994, p. 47-58.

14 Junto al manuscrito de la Historia riqueriana descubierto en Bamberg en 1833, están el de Wolffenbüttel y el de Leiden (que incluye también los textos conciliares de Saint-Basle, además de los de los concilios de Mouzon y de Reims).

15 Cf. M. MOSTERT, «Les traditions manuscrites des oeuvres de Gerbert», en Gerbert l'Européen, ed. cit. (1997), p. 307-325.

16 De hecho, el autor divide sù exposición en once puntos, pero uno de ellos («Gerberto y Otón III [996-999]») se solapa con dos de dichas etapas - la del arzobispado de Reims (7) y la del de Ravena (8) - y el otro es un apéndice final («Gerberto después de Gerberto»).

17 Cf. J. Bolós I Masclans, Repertori d'antropònims catalans, I, Barcelona 1994, p. 320s.

18 A la abadía de Saint Géraud dedicaba su artículo N. ChaRBonNel en Gerbert l'Européen, ed. cit. (1997), p. 53-77.

19 Cf. J. LECLERCQ, «Interpretazione gerbertiana della vita monastica», en Gerberto... (Bobbio 1985), p. 677-689.

20 Cf. F. UdiNA I MARTORELL, «Gerberto y la cultura hispánica: los manuscritos de Ripoll», en Gerberto... (Bobbio 1985), p. 35-50; y P. Riché, «Gerbert d'Aurillac en Catalogne», en Catalunya i França meridional a l'entorn de l'any mil, Barcelona 1991, p. 374-377.

21 Cf. M. ZimmermanN, «Un formulaire du Xe siècle conservé à Ripoll», en Faventia, 1992, p. 25-86, y «Formule de consécrations d'église», en Autour de Gerbert d'Aurillac, Chartres 1996, p. 27-35.

22 Cf. A. Pladevall i Font, Silvestre II (Gerbert d'Orlhac), Barcelona 1998, p. 33-40.

23 Cf. P. RichÉ, «Les églises de France occcidentale et de Lotharingie à l'époque de Sylvestre II», en L'Église de France et la Papauté (Xe-XIIIe siècle), Bonn 1993, p. 48-53. 
3. Primera estancia en Roma (970-972). Fue a Roma en compañía del obispo de Vic, Atón, y posiblemente asistió a la boda de Otón II y Teófana, de cuyo hijo —el futuro Otón III- sería en su momento preceptor. En cuanto al asesinato de Atón - ya de vuelta, a manos de los partidarios del obispo de Narbona, según unos; o en Roma, en agosto de 971, según otros-, parece que no pueden precisarse ni el lugar ni la fecha. ${ }^{24}$

4. En la escuela de Reims. Centro de cultura intelectual, ${ }^{25}$ Reims tenía una importante biblioteca (Boecio, Cicerón, el Pseudo Dionisio) de la que se sirviría Gerberto en el ejercicio de su docencia y el número de cuyos manuscritos ampliaría considerablemente durante la veintena de años que duró allí, en dos etapas, su actividad. ${ }^{26}$ En la cuarta parte nos referiremos a las disciplinas que impartió; digamos aquí que tuvo gran éxito, pues además de ser sabio era un buen pedagogo, que ponía a disposición de sus alumnos instrumentos nuevos. Pero de entre los tenidos por discípulos suyos, un Fulberto de Chartres sólo le conoció a través de sus obras. ${ }^{27}$ En cualquier caso, el éxito suscita rivalidades, y Oterico de Magdeburgo le retó a una disputatio en Ravena sobre la división de las materias filosóficas. ${ }^{28}$.

5. Abad de Bobbio (982-983). El triunfo que había obtenido en Ravena debió de influir en el nombramiento de Gerberto como abad de Bobbio: Otón II aprovecharía el querer premiarle por aquél para confiar uno de los grandes monasterios italianos a quien pudiera reformarlo. ${ }^{29} \mathrm{~A}$ las envidias que la importancia del lugar suscitaba y a las críticas contra el mismo por parte de ciertos personajes -incluída la emperatriz madre, Adelaida - el nuevo abad no dudó en responder con firmeza, pese a que se consideraba el más fiel vasallo de los otones. De esta época sería, por lo demás, la primera de sus Cartas; y la elaboración del catálogo de la biblioteca de Bobbio acaso se debiera a su iniciativa. ${ }^{30}$

6. De nuevo en Reims. Al trabajo docente que prosiguió al volver a Reims, Gerberto añadiría una actividad política que queda mejor reflejada en sus Cartas que en la Historia de Riquerio: fue secretario de Adalberón, arzobispo de Reims; ${ }^{31}$ ayudó a la emperatriz Teófana, viuda, a salvar el trono de su hijo, el futuro Otón III ; ${ }^{32}$ en 987, contribuyó a que Hugo Capeto accediera al trono francés, y luego ejerció de secretario suyo en sus relaciones epistolares tanto con el conde Borrell, a las que ya se ha aludido, ${ }^{33}$ como con el emperador de Bizancio; pese a no ser nombrado él mismo sucesor, al morir en 989 el arzobispo, no dudó en ponerse al servicio de Arnulfo, el designado; y parece que en alguna ocasión relativizó la legitimidad de los capetos («ínterim del poder real»), no reconocidos por los señores del Midi francés. ${ }^{34}$ En fin, su papel en el concilio de Saint-Basle — cuyas actas redactó- fue, para unos, el de puro y pasivo testimonio, mientras que, para

24 Cf. P.H. FreEDman, The Diocese of Vic, New Jersey 1983, p. 20.

25 Cf. J. DevisSE, Hincmar archevêque de Reims 845-882, 3 vol., Ginebra 1976.

26 Cf. P. RichÉ, «La bibliothèque de Gerbert d'Aurillac», en Mélanges de la Bibliothèque de la Sorbonne, París 1948, p. 94-103 (reimpr. en 1993 en Éducation et culture dans l'Occident médiéval), y «L'enseignement de Gerbert à Reims dans le contexte européen», en Gerberto... (Bobbio 1985), p. 52-54; y J. VEZIN, «Une quète de classiques: un Cicéron copié pour Gerbert», en Autour de Gerbert d'Aurillac, ed. cit. (1996), p., 276-283, y «La production et la circulation des livres dans l'Europe du Xe siècle», en Gerbert l'Européen..., ed. cit. (1997), p. 205-218.

27 Sobre la relación entre las escuelas de Reims y Chartres, cf. AA. Vv., Le temps de Fulbert de Chartres, Chartres 1996.

28 Cf. RICHER, Histoire de France, ed. cit., III, p. 58-65.

29 Cf. P. RicHÉ, «ll y a mille ans: Gerbert d'Aurillac en Italie du Nord», en Byzantinische Forschungen, 1987, p. $473-484$.

30 Cf. M. TosI, «Il governo abbaziale de Gerberto a Bobbio», en Gerberto... (Bobbio 1985), p. 197-234; y J.P. GENEST, «Inventaire de la bibliothèque de Bobbio», en Autour de Gerbert d'Aurillac, ed. cit. (1996), p. 250-260.

31 Sobre éste, cf. M. BuR, «Adalbéron de Reims reconsidéré», en Le roi de France et son royaume autour de l'an mil, París 1992, p. 55-64.

32 Sobre Teófana, cf. AA. Vv., Abendländische Buchkunst zur Zeit der Kaiserin Theophanu, Colonia 1991, y The. Empress Theophanu, Cambridge 1995.

33 Cf. supra epígrafe 2.- Estudiante en Cataluña.

34 Cf. Y. SASSIER, Hugues Capet, París 1987, p. 227; y J. Dufour, «Obédience respective des Carolingiens et des Capétiens (fin Xe siècle - début Xe siècle)», en Catalunya i França meridional a l'entorn de l'any mil, ed. cit. (1991), p. 21-44. 
otros, habría sido el de activo inspirador ${ }^{35}$ aun en el segundo caso, de lo que se trataría quizás fuese de legitimar a Hugo Capeto más que de juzgar a un arzobispo traidor. ${ }^{36}$

7. Arzobispo de Reims (991-997). Su conflicto con Roma ha sido muy estudiado, por razones polémicas, por parte de galicanos y protestantes: ${ }^{37} \mathrm{y}$ - siguiendo el ejemplo de Yves Congar - cabe siempre matizar. $^{38}$ En cualquier caso, la selección de las cartas de entonces que él mismo ordenó que fuesen conservadas puede servir de modelo en lo pastoral. Por lo demás, en primavera de 997 se dirigiría a la corte de Magdeburgo, donde se hallaba Otón III - a cuya coronación en Roma el año anterior tampoco había faltado (habiéndole redactado entonces incluso algunas cartas) -; y, estando allí, recibió el encargo de construir un horologium, al que luego nos referiremos. Para compensarle de la pérdida de la sede de Reims, el papa Gregorio V, primo del emperador, y éste le asignaron la de Ravena.

8. Arzobispo de Ravena. El papel desempeñado por Gerberto en su nuevo destino fue ciertamente activo. ${ }^{39}$ Participó además en el concilio de Pavía, convocado por el emperador, y en el de Roma, de principios de 999.40

9. Papa Silvestre II (999-1003). Las bulas de su pontificado - publicadas por Harald Zimmermann en 1985- permiten conocer la acción de Silvestre II en la cristiandad. Ya nos hemos referido a las dirigidas a sus viejos amigos catalanes; ${ }^{41}$ intervino asimismo en Francia y Lotaringia, ${ }^{42}$ e hizo saber al emperador sus ideas políticas. ${ }^{43}$ Con Otón III creó, en fin, las iglesias de Polonia y Hungría. ${ }^{44}$ Su epitafio puede verse en San Juan de Letrán. ${ }^{45}$

Llamado el «Fausto medieval», Gerberto fue objeto, a partir del s. XII, de desastrosas leyendas; ${ }^{46}$ e incluso hoy no deja de inspirar novelas pintorescas, de escaso valor histórico. ${ }^{47}$

[4] Lo que de Gerberto más nos interesa es su aportación a la historia cultural de Occidente, es decir, la que fuera su actividad intelectual. Los trabajos más recientes sobre dicha aportación pueden agruparse en torno a los cuatro más significativos aspectos de esta su actividad intelectual.

35 Cf. G. GIORDANENGO, «Le concile de St.-Basle. Actes conciliaires redigés par Gerbert», en Autour de Gerbert d'Aurillac, ed. cit. (1996), p. 134-140.

36 Cf. V. HuTH, «Erzbischof Arnulf von Reims und der Kampf um das Königtum im Westfrankenreiche», en Francia, 1994, p. 85-124.

37 Cf. E. BuRY, «Gerbert et l'historiographie gallicane: de la philologie au débat des idées», en Gerbert l'Européen..., ed. cit. (1997), p. 325-333; y B. DompIER, «La controverse protestante et Gerbert (XVIe-XVIIIe siècle)», id., p. 335-346.

38 Cf. Y. ConGAR, L'ecclésiologie du haut Moyen Âge, París 1968, p. 183 («Entre Gerberto y Silvestre II hay un contraste. Pero no hay que perder de vista que uno y otro son el mismo hombre, animado por el sentido de la unidad de la Iglesia»); y M. SoT, «Le moine Gerbert; l'église de Reims et l'église de Rome», en Gerbert l'Européen..., ed. cit. (1997), p. 135-149.

39 Cf. A. VASINA, «Gerberto arcivescovo di Ravenna», en Gerberto... (Bobbio 1985), p. 255-271.

40 Cf. P. Riché, Les grandeurs de l'an mille, París 1999, p. 260-263.

41 Cf. supra epígrafe 2.- Estudiante en Cataluña.

42 Cf. P. RICHÉ, artíc. cit. supra nota 23; H. ATSMA, «Le pape et la discipline ecclésiastique: lettre de Silvestre II a l'abbé Odilon de Cluny», en Autour de Gerbert d'Aurillac, ed. cit. (1996), p. 178-183; y O. GuYOTJEANNIN, «Une élection épiscopale contestée: privilège de Silvestre II pour l'évêque de Puy», en id., p. 170-176.

43 Cf. L. Hoffmann, Gerbert von Reims und die Idee der «Renovatio Imperii Romanorum, Munich 1988 (memoria de investigación dactilografiada).

44 Cf. P. RIcHÉ, Les grandeurs de l'an mille, París 1999, p. 293-304.

45 Cf. R. FAVREAU, «Le souvenir officiel: l'épitaphe de Silvestre II à St. Jean de Latran», en Autour de Gerbert d'Aurillac, ed. cit. (1996), p. 336-340.

46 Cf. M. OLDoNI, «Gerberto e la sua Storia», en Studi Medievali 18 (1977), p. 629-704, y «A Fantasia dicitur fantasma», id. 20 (1979), p. 1-207; y P. Riché, Gerbert d'Aurillac le pape de l'an mil, ed. cit. (1987), p. 9-15 («Des légendes à l'histoire») i 259.

47 Cf. R. Sussan, Les renégats de l'an mil, París 1992; D. Davidenko, Le pape de l'an mil, París 1993; y CH. RaYmo, Dans les serres de Faucon, París 1994. 
a) Lo literario. El estudio de autores clásicos le dio a Gerberto un latín excepcional comparado con el de sus contemporáneos ${ }^{48}$ obviamente, no ajeno al de la Vulgata ni al de la tradición patrística. ${ }^{49}$ Tanto los epitafios conservados en su correspondencia o su poema sobre Boecio, como el himno sobre la Sabiduría o un acróstico parisino que se le atribuyen, parecen hablarnos de un literato en ciernes. ${ }^{50}$

b) El «trivium». Dejando la gramática para otro maestro, Gerberto explicó en Reims a sus alumnos los autores clásicos, sirviéndose de Quintiliano y Cicerón en retórica. ${ }^{51}$ Pero fue la dialéctica lo que le dio fama de «filósofo»; y su De rationali et ratione uti es una «obra insólita» que anticipa las «cuestiones disputadas de la época escolástica». ${ }^{52}$

c) El «quadrivium». Conocedor no sólo de los tratados filosóficos de Boecio sino también de sus traducciones introductoras de la ciencia griega en Occidente, Gerberto tuvo acceso en su estancia en Cataluña a las traducciones de los sabios árabes. Títulos ya clásicos y recientes coloquios han versado sobre las cuatro disciplinas que integraban lo que pudiera llamarse - dejada aparte la medicina - la ciencia medieval, refiriéndose en general a su estado en dicha época o a Gerberto, concretamente. ${ }^{53}$ No faltan, por supuesto, los escritos sobre el conocimiento gerbertiano de las matemáticas (aritmética y geometría); ${ }^{54}$ pero aún se sigue debatiendo hasta qué punto dominaba Gerberto los números árabes. Objeto también de discusión es el alcance de su saber astronómico: el horologiun que se le encargara en Magdeburgo ${ }^{55}$ sería un nocturlabio ${ }^{56}$ que Gerberto conociera la obra ptolomaica no pasa de ser posible $;{ }^{57}$ y no todo son coincidencias acerca de la autoría del comentario sobre el astrolabio a él atribuido. ${ }^{58}$ En cuanto a la música -el tratado boeciano sobre la

48 Cf. A. BROMET, «Étude sur la langue de Gerbert», en Revue de la Haute Auvergne, 1947-1949, p. 217-230, y 1952-1953, p. 91-109; y J.-P. CALLU, «Introduction», en Correspondance de Gerbert d'Aurillac, ed. cit. (1993), p. XxxIIxxxrv, y «Les mots de Gerbert», en Gerbert l'Européen, ed. cit. (1997), p. 151-167.

49 E. RorTENBURGER, Gerbert and the Classics, Cincinnati 1964; y D.V. DAMME, «Gerbert et la culture patristique», en Gerberto... (Bobbio 1995), p. 691-693.

50 Cf. T.E. MoEHS, «Gerbert of Aurillac as Link between Classicism and Medieval Scolarship», en Gerberto... (Bobbio 1995), p. 331-349; y P. BouRGaIN, «La création poétique: l'hommage de Gerbert à Boèce», en Autour de Gerbert d'Aurillac, ed. cit. (1996), p. 296-298. El poema sobre la sabiduría fue recogido en el vol. V de los Monumenta Germaniae Historica Poet. Ev. carol., p. 475; y en cuanto al mencionado frontispicio, cf. C.W. BROCKETT, «The Frontispiece of Paris ms. latin 776 Gerbert's acrostic pattern poems», en Manuscripta, 1995, p. 3-25.

51 Cf. J. VÉzIN, artíc. cit. supra nota 26.

52 Cf. C. Frova, «Gerberto philosophus. Il De rationali et ratione uti», en Gerberto... (Bobbio 1995), p. 351-377; y D. PoIREL, «L'art de la logique: le De rationali et ratione uti de Gerbert», en Autour de Gerbert d'Aurillac, ed. cit. (1996), p. $312-320$.

53 Cf. G. Beaujouan, «L'enseignement du Quadrivium», en Settimale di Spoleto, 1972, reimpr. en Par raison de nombres (Variorum Reprints), Aldershot 1991, vol. I; U. LINDGREN, Gerbert von Aurillac und das Quadrivium, Wiesbaden 1976; y W. BERGMANN, Innovationen im Quadrivium des 10. und 11. Jahhunderts. Studie zur Einfiihrung von Astrolab und Abakus im lateinischen Mittelalter, Stuttgart 1985. Cf. también las Actas, en curso de publicación, del congreso de Chieti (mayo de 1996) La matematica e il suo enseignamento in epoca medievale y del de Avranches-Mont-St.-Michel (septiem-
bre de 1996) Science antique, science médiévale.

54. Cf. C. Frova, «Le opere aritmetice di Gerbert d'Aurillac», en Miscellania Morghen, vol. I, Roma 1974, p. 331; G. BEAUjOUAN, «Les Apocryphes mathématiques de Gerbert», en Gerberto... (Bobbio 1995); p. 645-658, y «Les chiffres arabes selon Gerbert: l'abaque du Pseudo-Boèce», en Autour de Gerbert d'Aurillac, ed. cit. (1996), p. 325; K. VoGeL, «L'Aritmetica et la Geometria di Gerberto», en Gerberto... (Bobbio 1995), p. 577-596; y M. FoLKERTS, «Frühe Darstellungen des Gerbertschen Abakus», en DD. AA., Itinera mathematica, Siena 1996, p. 23-43.

55 Cf. supra epígrafe 7.- Arzobispo de Reims.

56 Cf. E. PoulLE; «L'astronomie de Gerbert», en Gerberto... (Bobbio 1995), p. 597-617.

57 Cf. U. LindGREN, «Ptolomée chez Gerbert d'Aurillac», en Gerberto... (Bobbio 1995), p. 619-644.

58 Cf. J.M. Millàs VAllicrosa, Assaig d'història de les idees físiques i matemàtiques a la Catalunya medieval, Barcelona 1931, p. 656-658; A. BonST, Astrolab und Klosterreform an der Jahrtausenswende. Heidelberg 1989; G. BEAUJOUAN, «Les Apocryphes mathématiques de Gerbert», en Gerberto... (Bobbio 1995), p. 656-658; DD. AA., The oldest latin astrolabe, número especial de Physis (Florencia), 1995; M. MOSTERT, «Les traditions manuscrites des oeuvres de Gerbert», en Gerbert l'Européen, ed. cit. (1997), p. 317. 
cual comentó-, él mismo construyó, en fin, un órgano, además de escribir un tratado sobre la medida de sus tubos. $^{59}$

d) La medicina. Pese a no enseñarla -la medicina no formaba parte del quadrivio-, Gerberto conoció dicha disciplina: su discípulo Riquerio había ido a Chartres a consultar los manuscritos hipocráticos, él mismo leyó manuscritos de medicina y sus cartas revelan un interés por este arte, aunque no fuese especialista en él. ${ }^{60}$

Brevemente: Gerberto fue un humanista que sorprendió y hasta escandalizó a ciertos miembros del clero de su tiempo ${ }^{61}$ En este sentido sería un representante de lo que Riché ha llamado el «tercer renacimiento carolingio», contra el que reaccionarían Pedro Damián y otros antidialécticos de la primera mitad del s. XI (opuestos a quienes afirmaban que «todas las palabras de la Escritura habían de ser sometidas a la autoridad de la dialéctica» y que solían testimoniar «más confianza en Boecio que en los autores sagrados»). Precisamente como Boecio - que, tras su proceso, fue tildado de mago por quienes no entendían el alcance de su saber-, Gerberto sería también a partir de fines del s. XI legendariamente revestido de magia.

\section{OTRAS PONENCIAS Y COMUNICACIONES: APORTACIONES PUNTUALES}

De carácter predominantemente histórico, el interés filosófico-cultural del Congreso se adscribía fácilmente a sólo unas pocas de sus ponencias y comunicaciones. A ellas se ceñirá el resto de la presente nota, pero su selección no supone valoración alguna comparativa respecto a las otras numerosas aportaciones congresuales dejadas aquí de lado.

[A] La primera de las ponencias que queremos reseñar fue la de Thomas N. Bisson, de la Universidad de Harvard, relativa a la tradición magisterial hacia el año mil y centrada en la comparación de la actividad docente de Gerberto en Reims con la de Abbón de Fleury y la de Fulberto de Chartres. ${ }^{62}$ A diferencia de la inaugural de Riché - acaso aséptica en exceso (aunque semejante carácter encajase bien con el hecho de ser pura presentación del «estado de la cuestión» gerbertiana en la actualidad)—, la ponencia de Bisson sonaba a apasionada y comprometida, aunque sus conclusiones, además de ser sorprendentes, pudiesen acabar dejando una cierta insatisfacción; ${ }^{63}$ en cualquier caso $-\mathrm{y}$ por deseo explícito del autor-, el texto inglés de su aportación había sido traducido al catalán para ser publicado en dicha lengua en las Actas, que los asistentes al Congreso podían tener en sus manos al escuchar al ponente. ${ }^{64}$ En cuanto a lo que éste pretendía, lo enunciaba él mismo de entrada: exponer tanto la importancia como la específica peculiaridad del aprendizaje en la enseñanza medieval, considerando la naturaleza de las relaciones entre estudiante y maestro.

Gracias a los trabajos de Ramon Ordeig —así homenajeó Bisson a quien, allí presente, había de hablar tras él- podemos ya saber que el conde Borrell encomendó al joven Gerberto a Atón porque éste, antes de lle-

59 El tratado, Mensura fistularum, fue editado por K.J. Sachs (Stuttgart 1970-1980). Sobre Gerberto y la música, cf. K.J. SACHS, «Gerbertus cognomento musicus», en Archiv fïr Musikwissenschaft, 1972, p. 257-274; M. HuGLO, «Gerbert of Reims», en New Grove's Dictionary of Musik and Musicians, 1980, vol. VII, p. 250; y C. MEYER, «Gerbertus musicus. Gerbert et le fondements du système acoustique», en Gerbert l'Européen, ed. cit. (1997), p. 183-191.

60 Cf. D. JACQUART, «La médicine au Xe siècle», en Gerbert l'Européen, ed. cit. (1997), p. 219-231; y M. VAZQUEZ BUJAN, «La circulation des manuscrites hippocratiques», en Id., p. 233-246.

61 Cf.P. RichÉ, «Les conditions de la production littéraire: maitres et écoles», en Mittellateinisches Jahrbuch 24/25 (1989-1990) p. 413-4221; «L'Humanisme au Xe siècle», en Gli umanesimi medievali, Florencia 1998, p. 551-558; y Les grandeurs de l'an mille, París 1999, p. 167-220 («La troisième renaissance carolingienne»).

62 Cf. T.N. BIsson, «L'aprenentatge dels savis a Europa pels volts de l'any mil: les lliçons de Gerbert, Abbo i Fulbert», en Actes del Congrés Internacional: Gerbert d'Orlhac i el seu temps, ed. cit., p. 577-591.

63 A diferencia también de las páginas anteriores (abundantes en notas, reflejo del estado bibliográfico de la cuestión), las que siguen se ahorrarán casi del todo el aparato crítico.

64 Cf. Actes del Congrés.... ed. cit., p. 577, nota 1, en la que, además de dejar constancia de dicho explícito deseo del autor, la traductora consigna asimismo el título original de la ponencia: «Learned Apprenticeship in Europe towards the year 1000: the Lessons of Gerbert, Abbo, and Fulbert». 
gar a obispo de Vic (957-971), había sido archidiácono de Gerona, cuyo obispo Gotmar (944-954) se había interesado especialmente por la enseñanza que se daba en tierras musulmanas, y él mismo había viajado a Córdoba, posiblemente con algunos discípulos a los pudo conocer más tarde Gerberto. ${ }^{65}$ Enviado a Vic para recibir una sólida formación - no simplemente para ir a la escuela—, el joven monje venido de Aurillac conoció junto a Atón un muy buen aprendizaje en matemáticas, pero no tanto en la ciencia lógica, según consignó Riquerio en su Historia de Francia, en la que nada dijo ni sobre el método de enseñanza del obispo ni sobre la opinión de Gerberto sobre el particular. Parece ser que éste se sirvió sobre todo de una lectura personal de libros orientada por Atón, y no tanto del mismo magisterio del obispo (demasiado comprometido por entonces en el intento de conseguir para su diócesis el estatuto de sede metropolitana). Precisamente recordando libros manejados en aquellos tiempos —en Vic, en Ripoll y en Gerona- Gerberto escribiría años más tarde a amigos catalanes (Miró Bonfill o Llobet de Barcelona), desde Reims o desde Bobbìo, pidiéndoles que le enviasen una determinada obra sobre aritmética o aquella otra traducción de cierto escrito sobre astrología, respectivamente.

Sea del tema lo que sea, de retorno de Roma Gerberto no sintió necesidad de volver ni a Aurillac ni a pasar los Pirineos, y prefirió completar su instrucción en Reims, con la perspectiva de un reputado maestro y de libros relativos al ámbito de la dialéctica: es decir, de aquella lógica en la que se sentía más bien flojo. Y es que lo que buscaba era alcanzar los límites del conocimiento, comprendiendo la totalidad y las conexiones de todas las materias. Ahora bien: de alumno destacado no tardó en pasar a ser sustituto de su mismo maestro, Geranno, siendo nombrado para enseñar «las artes» a «las masas de estudiantes» que allí se reunían y entre los cuales se hallaba Riquerio. Por él sabemos el orden en que Gerbeito explicaba la nueva ciencia en la que se había consolidado hacía poco: empezaba con la Isagogé de Porfirio, pasaba luego por las Categorías y el De interpretatione aristotélicos y, en clases más avanzadas, seguía con la dialéctica y la retórica, basándose en la lectura y el comentario de los poetas (Virgilio, Estacio, Terencio, Horacio); en cuanto a las matemáticas, Riquerio evoca a Gerberto como maestro pionero que desarrollaba instrumentos de medida y cálculo y enseñaba — como nadie hasta entonces - teoría musical. ${ }^{66}$ «Hombre de gran genio y admirable elocuencia» que, «como antorcha encendida», iluminaba «toda Francia» - según. Riquerio-, Gerberto fue reconocido por maestros como Abbón de Fleury, también antiguo alumno de Geranno, o sería retado a confrontación pública por otros, como hizo Otrico de Magdeburgo; e incluso la tradición lo presentó como maestro del gran Fulberto de Chartres. No es, pues, raro que hubiera sido promovido como abad de Bobbio, monasterio benedictino de espléndida biblioteca pero que no había conseguido retenerle más de un año.

La tercera y última parte de la ponencia —que en las Actas ocupa más de la mitad de las páginas del conjunto- se centraba en comparar el magisterio gerbertiano en Reims con el de Abbón en Fleury y el de Fulberto en Chartres. Algunas de las conclusiones finales son quizás de particular interés para nosotros, por su carácter de sorprendentes y pese a que puedan acabar aportando una cierta decepción. En los cincuenta años que median entre la época del maestrazgo de Gerberto en Reims y la del de Fulberto de Chartres, la escena de la enseñanza había cambiado algo: había más libros, más maestros en artes y más clérigos jóvenes y monjes en busca de aquéllos y de éstos; y ningún estudioso tuvo que repetir en el s. XI la búsqueda de libros científicos en la que se había aventurado Gerberto. Pero sería un error exagerar el cambio que se había operado al respecto: no todas las bibliotecas carolingias tenían todos los libros importantes, la enseñanza seguía consistiendo en aprender de libros escritos siglos atrás y algunos de éstos - como el de las Categorías y el De la interpretación, de Aristóteles, en versión de Boecio- ${ }^{67}$ debían de resultar a los estudiantes de entonces, y así lo siguen pareciendo a los de hoy, bastante duros. Lo característico del aprendizaje en el s. X era aquella necesi-

65 El ponente se refería (como consignó en nota) al artículo de R. ORDEIG I MATA, «Ató, bisbe i arquebisbe de Vic (957-971), antic arxiprest-ardiaca de Girona», en Studia Vicensia, 1 (1989), p. 61-97; y declinaba seguir hablando del maestro de Gerberto, pues de él había de tratar la ponencia siguiente: R. ORDEIG I MATA, «Ató de Vic, mestre de Gerbert d'Orlhac», en Actes del Congrés..., ed. cit., p. 593-618, de la cual queremos hacer particular mención aquí, aunque no hayamos de referirnos a ella en estas páginas por referirse más a Atón que a Gerberto.

66 La música seguía siendo entonces inseparable - como ya lo era desde los pitagóricos y Platón- de la matemática: ni que fuese por aquello de que la nota musical depende de la medida de la cuerda...

67 Cf. Migne, PL 64, col. 156-640. 
dad compulsiva de un guía en la lectura que sentían hasta los estudiantes mejor dotados: Berenguer de Tours lo encontraría en Fulberto de Chartres, como Gerberto lo había hallado en Atón de Vic; y a finales del s. XI seguía existiendo la misma fascinación por aprender de un maestro, dado el escaso número que aún había de ellos. La enseñanza no era todavía académica, sino que creaba unos vínculos personales que frecuentemente resultaban inseparables de las verdades propuestas y de los análisis realizados. En este sentido, la relación entre estudiante y maestro suena como una corriente de intercambio mutuo y como reconocimiento del poder en formas de dependencia personal. El de las generaciones de discípulos de Gerberto, Abbón de Fleury y Fulberto fue un tiempo en que los nuevos señoríos se extendían por toda la cristiandad, y especialmente en la Iglesia. ¿No fue el aprendizaje gerbertiano un reconocimiento de dependencia respecto de Atón de Vic?; ¿no fue «confiado», en Reims, Gerberto a Geranno?; ¿no seguían los estudiantes de Magdeburgo con celosa fidelidad a su maestro Otrico durante su disputa con Gerberto?; ¿y la devoción de sus discípulos hacia Fulberto no reflejaba la existencia de un quasi señorío de éste sobre ellos? Gerberto en Reims, Abbón en Fleury y Fulberto en Chartres coincidieron en entender los estudios como un todo, e incluso se ha dicho que lo restablecieron; ahora bien, el progreso que en ellos consiguieron fue frágil, y acaso ilusorio.

[B] Ya hemos aludido a la segunda ponencia, de Ramon Ordeig, que integraba - junto con la que acabamos de resumir - el cuarto grupo temático congresual, relativo a la enseñanza en el año mil ${ }^{68}$ Pero quisiéramos aquí mencionar también una de las tres comunicaciones que las acompañaban, y de la que las Actas sólo ofrecen el resumen, pues el texto debió de retrasarse como para no ser publicado en ellas. Obviamente, ni nos referimos a ella por no haber sido publicada ni podemos, en consecuencia, ofrecer extracto alguno de su contenido; mas queremos dejar constancia del interés que el texto hubiera tenido, de haber visto la luz. Su título rezaba: «Formación cultural de Gerberto de Aurillac en la Marca hispánica»; ${ }^{69}$ y correspondía a un resumen de la segunda parte del trabajo de licenciatura presentado en 1947 en la Gregoriana por Jaume Tarracó i Planas e intitulado «Formación cultural de Gerberto (Silvestre II) en San Geraldo de Aurillac y en la Marca Hispánica». ${ }^{70} \mathrm{El}$ interés del texto habría sido doble: por un lado - supuesto el tema mismo-, intrínseco; y extrínseco, por otro, dado que la exposición de lo conocido al respecto hace más de medio siglo hubiera permitido una elocuente comparación con el que es èl estado actual de la cuestión.

[C] El conocido historiador y paleógrafo Anscari M. Mundó estructuraba su ponencia, la segunda $-\mathrm{y}$ la que más nos puede interesar aquí- de las cuatro que integraban el penúltimo grupo temático del Congreso, relativo a la ciencia y la cultura, a partir de un minucioso análisis de las inscripciones grabadas en el astrolabio carolingio de la colección Marcel Destombes, actualmente en el Institut du Monde Arabe, de París. ${ }^{71}$ En su exposición, el ponente confirmaba con certeza la procedencia y la fabricación catalanas de dicho astrolabio, datable entre el tercero y el último cuarto del $\mathrm{s}$. X; de esta manera, corroboraba que la latitud grabada en uno de los tímpanos de la pieza (41³0' ROMA ET FRANCIA) correspondería al área del condado de Barcelona. Es conocida la gran relación intercultural existente entre los condados catalanes y el mundo árabe del resto meridional de la Península, mundo en el que había unos avanzados conocimientos matemáticos y astronómicos. Por lo demás, hubo de ser importante al respecto el papel jugado por Seniofred Llobet, archidiácono de Barcelona y traductor al latín de libros de astronomía árabes, a quien Gerberto conoció durante su estancia en tierras catalanas. Tanto uno como otro coinciden, por época y por conocimientos, con la construcción del astrolabio en cuestión, el cual pudo haber sido llevado a Francia, donde se encuentrā hoy, por Gerberto mismo en uno de sus viajes posteriores.

\section{Cf. supra nota 65.}

69 Cf. Emília TaRracó I Planas, «Formació cultural de Gerbert d'Orlhac a la Marca Hispànica», en Actes del Congrés..., ed. cit., p. 635s.

70 Es de suponer que la titular de la comunicación (Emília) es hermana del autor (Jaume) del trabajo de investigación que se trataba de exponer abreviadamente, a modo de homenaje al mismo (posiblemente ya fallecido). En el resumen publicado del texto no incluido en las Actas se indican los cuatro capítulos de que constaba la segunda parte en cuestión de aquel trabajo: «1. Maestros de Gerberto en la Marca Hispánica; 2. Lugar de los estudios de Gerberto, deducido de las fuentes de sus obras; 3 . Estudios de Gerberto en la Marca Hispánica y su alcance; y 4. Conclusiones».

71 Cf. A.M. Mundó, «Entorn de l'astrolabi de Gerbert», en Actes del Congrés..., ed. cit., p. 665-676, cuyo mismo resumen inicial casi nos limitamos a traducir en este nuestro. 
[D] La última de las cuatro comunicaciones adjuntas a las ponencias del mencionado grupo temático congresual sobre ciencia y cultura volvía a despertar nuestro interés, y a su vez ofrece peculiaridades parecidas a las de la otra comunicación antes recensionada. Del interés del tema que había de tratarse en ella daba testimonio su título: «Gerberto y la introducción decimal indo-árabe en el Occidente cristiano: el Liber abaci»; ${ }^{72}$ pero, tras él, en las Actas se añadía una cruz al nombre del autor: Jean Cassinet $\nmid{ }^{73}$ Sea, pues, el resumen que él mismo enviara el que nos dé cuenta de la comunicación de la que su muerte nos privó.

«Cuando Gerberto vino a estudiar a Cataluña en 967 bajo la égida bien conocida de Atón, obispo de Vic, entre los tesoros desconocidos de la ciencia árabe debía de descubrir la numeración decimal de posición, la que hoy utilizamos y que los árabes trajeron de la India. El nuevo impulso dado por Gerberto al uso del ábaco -un tablero de columnas con el que se podían hacer las cuatro operaciones elementales de la aritmética mediante fichas - le permitió introducir un sistema decimal de posición. Para efectuar la más delicada de las operaciones, la división, Gerberto hubo de inventar un procedimiento, el de la división «por diferencia», muy riguroso e ingenioso, y tan rápido como los nuestros habituales. Fueron sobre todo sus discípulos quienes transmitieron, desde finales del s. X, sus fórmulas, y muy especialmente Bernelino en su Liber abaci, que pronto será publicado en edición bilingüe latino-francesa y una excelente copia de cuyo manuscrito se halla en la biblioteca de la Escuela de medicina de Montpellier. Aunque la empresa gerbertiana no tuviera un éxito inmediato - habría que esperar a fines del s. XII a que la numeración decimal de posición se impusiera con fuerza en el Occidente cristiano-, lo cierto es que el maestro supo atraer la curiosidad de los hombres de sabiduría occidentales, impulsándoles a tomar contacto con la matemática árabe y a crear, así, las condiciones para posteriores progresos».

[E] La última de las aportaciones al Congreso a la que hemos de referirnos para acabar estas páginas fue también la última de las ponencias del mismo, con la que éste concluía solemnemente en Ripoll. Correspondía y servía de colofón al asimismo último de los grupos temáticos de las sesiones, centrado en el monacato y el papado en el año mil; corría a cargo de Walter Brandmüller, de la Canonica Vaticana, y versaba sobre «Silvestre II: el primado romano en el umbral del segundo milenio». ${ }^{74}$ No es que el tema del papado haya de interesar particularmente, de manera directa, a la reflexión histórico-filosófica. Pero la cuestión del primado - originada en la cristiandad del Imperio romano y consolidada, tras la caída de éste, a lo largo de la edad media- es asunto tanto o más político que estrictamente teológico o doctrinal; ${ }^{75}$ y es, a la vez, inseparable del

72 Cf. J. CASINET, «Gerbert et l'introduction de la numération décimalè arabo-indienne en Occident chrétien: le Liber abaci», en Actes del Congrés..., ed. cit., p. 726s.

73 La imprevista muerte del autor, miembro del Centre International d'Histoire des Sciences Occitanes, de la Université Toulouse-le-Mirail, justificaba, pues, en este caso que dichas Actas no ofrecieran más que el resumen de su aportación, sin el texto de la misma. Ahora bien: acaso adivinando que su vida no daría de sí como para permitirle leer su comunicación, Cassinet envió de ella un resumen tan bien hecho que nos permite hacernos perfectamente cargo del contenido que la misma hubiera aportado. Por eso, podemos volver a servirnos - con mayor rigor, aún, que en el caso de la ponencia anterior- de una traducción de dicho resumen para ofrecer el nuestro.

74 Cf. W. BRANDMÜLleR, «Sylvester II. Römischer Primat an der Schwelle zum 2. Jahrtausend», en Actes del Congrés..., ed. cit., p. 867-882.

75 E incluso puede decirse que contagia de determinaciones políticas a otros temas teológicos que, en sí mismos, pueden parecer del todo ajenos a cualquier politización. Es, en efecto, tanto o más política que estrictamente teológica 0 doctrinal, dado que la cuestión del primado romano remonta su ya antiguo planteamiento a algunos de los primeros grandes Concilios ecuménicos; ahora bien, de la decisiva inspiración política de dichos concilios nadie puede hoy en día dudar: no sólo eran convocados por el emperador y sus actas tan sólo sancionadas por el obispo de Roma, sino que éste negaba dicha sanción a uno u otro de los cánones conciliares que no se adecuasen a la convicción de que la sede romana tenía la primacía sobre otras, como Constantinopla o Alejandría (así hizo León I, en el 451, con el correspondiente canon de Calcedonia). El tema del primado era lo que, en el fondo, se debatía en Éfeso, ya en el 431, al condenar a Nestorio so pretexto de su oposición al theótokos («madre de Dios»); Alejandría conseguía con dicha condenación reducir la primacía del patriarcado constantinopolitano - cosa que no disgustaba en absoluto a Roma-, pero a la vez salpicaba políticamente la comprensión de un tema como el de la Encarnación, dejando tocada para siempre la denominación mariana: de ahí que hayamos dicho que, además de ser política, la cuestión del primado contagiaba de determinaciones políticas a otros temas teológicos que, en sí mismos, pudieran parecer del todo ajenos a cualquier politización. 
así llamado agustinismo político: una teoría sólo germinalmente enraizada en el obispo de Hipona, explícitamente tematizada con Carlemagno, fraguada en la pugna de las investiduras y consumada como choque de poderes entre Iglesia (de Roma) e Imperio (Romano-Germánico). De ahí que sea cuestión doblemente vinculada, de manera intrínseca, a la reflexión político-filosófica medieval; y de ahí, también, que la incidencia en ella de la exposición final del Congreso hubiera de despertar el interés por ésta en el autor de la presente nota.

El interés no quedó defraudado: Silvestre II quiso ejercer el primado, proclamándose summus et universalis papa, pero de hecho sólo lo hizo allí donde llegaba el poder de Otón III, el emperador al que le unía una gran amistad y de la mano del cual pudo emprender tareas como la de establecer una jerarquía eclesiástica en Hungría y Polonia. Su voluntad de ejercer el primado se manifiesta (en el que fue su primer documento papal) reponiendo en la sede de Reims al arzobispo Arnulfo al que él mismo sustituyera en 991, tras haber sido éste destituido - prescindiendo de la autoridad del entonces papa Juan XV - por el Sínodo de St. Basle de Verzy, convocado por un Hugo Capeto traicionado por el prelado al que él mismo había nombrado; y argumenta, como papa, que la destitución se había debido «a ciertos excesos» de quienes la decidieron (entre ellos, el propio Gerberto, quien en 995 se había visto también suspendido del cargo por decisión de otro sínodo: el de Mouzon). Sin embargo, su ejercicio del primado no tiene lugar alguno allí donde — como en el sur de Italiala autoridad de Otón III nada tiene que hacer frente al poder del emperador bizantino.

La peculiaridad del caso es que el papa del año mil, de más de sesenta años, y el emperador romano-germánico, de veinte años, tenían una muy peculiar buena relación. Con ella, el conflicto de poderes (entre Imperio e Iglesia) parece como que no se dé; y mediante ella, Silvestre II conseguía dar algunos pasos en favor de la libertad de la Iglesia frente al poder civil -romano, en el caso del papado; feudal, en el de diócesis y monasterios- que anticipaban la reforma gregoriana de casi un siglo después. En cualquier caso, su autoridad era concebida por este papa como inseparable de la pietas romana, de la liberalitas Ecclesiae y de la benignitas apostolica. De ahí que en su pontificado tuvieran un papel decisivo los sínodos (convocados por él mismo, y no tanto para imponer resoluciones cuanto para buscar en común soluciones), así como los legados y las cartas.

Josep Manuel Udina i Cobo Universitat Autònoma de Barcelona Campus de Bellaterra (Cerdanyola) 08193 Bellaterra 